\title{
Unilateral pleural effusion in chronic renal insufficiency: A clinician's challenge
}

\author{
Sudhir Kumar Atri', Karthik Ambalavana ${ }^{2}$, Anjali Dhanda ${ }^{2}$, Shiv Kumar ${ }^{2}$ \\ From ${ }^{1}$ Senior Professor, ${ }^{2}$ Postgraduate Student, Department of General Medicine, Pandit Bhagwat Dayal Sharma Post Graduate Institute of Medical \\ Sciences, Rohtak, Haryana, India
}

\begin{abstract}
Pleural effusion is a common clinical presentation of chronic kidney disease (CKD). Pleural effusion secondary to hypervolemia is usually bilateral in nature. We herein report a rare presentation of unilateral massive exudative pleural effusion in a 66-year-old male patient who is a known case of CKD, presented to us with severe dyspnea. On further evaluation, it was found that dyspnea was secondary to massive pleural effusion. After excluding other causes of pleural effusion, the patient was started on renal replacement therapy following which the patient improved markedly, and hence, pleural effusion was considered likely to be secondary to uremia. Uremia as such is a rare cause of pleural effusion and it should be considered as a diagnosis of exclusion.
\end{abstract}

Key words: Chronic kidney disease, Pleural effusion, Uremia

$\mathrm{C}$ hronic kidney disease (CKD) is a condition that presents usually with fluid overload. Pleural disease is a common problem in patients with chronic renal insufficiency which may be attributed to many etiologies, including congestive heart failure, infection, the presence of diseases associated with renal and pleural manifestations (e.g. systemic lupus in erythematosus), uremic pericarditis, malignancies, and pulmonary embolism. Unilateral pleural effusion is a diagnostic challenge in CKD patients. Uremic pleuritis results from an unknown putative agent, and therefore, uremic pleuritis is a diagnosis of exclusion [1].

\section{CASE REPORT}

A 66-year-old male presented to us with a complaint of shortness of breath for 4 days. It was not associated with chest pain, decreased urine output, vomiting, altered sensorium, fever, palpitations, joint pain, cough with sputum, night sweats, and weight loss. There was no history of connective tissue disease such as rheumatoid arthritis (RA). The patient is a known case of type 2 diabetes (for 22 years), hypertension (for 22 years), and CKD (3 years). He was on tab. amlodipine $10 \mathrm{mg}$ OD, prazosin $10 \mathrm{mg}$ OD, torsemide $20 \mathrm{mg} \mathrm{BD}$, and linagliptin $5 \mathrm{mg}$ OD. For the past 3 years, the patient was on regular medication and his renal functions remained stable, not requiring renal replacement therapy.

On admission, blood pressure was 160/100 $\mathrm{mmHg}$, pulse rate was $110 / \mathrm{min}$, and tachypneic at rest with a saturation of $85 \%$ at

\section{Access this article online}

Received - 21 April 2021

Initial Review - 06 May 2021

Accepted - 29 May 2021

DOI: $10.32677 /$ IJCR.2021.v07.i06.006 room air. On general physical examination, the patient was pale and having bilateral pedal edema. Icterus, clubbing, cyanosis, and jugular venous distension were absent. Respiratory system examination revealed tracheal deviation towards the right, dull percussion notes, and reduced air entry on auscultation over the left lung field. Cardiac examination was unremarkable.

Renal function tests performed 6 months back revealed creatinine $5.3 \mathrm{mg} / \mathrm{dl}$ and urea $152 \mathrm{mg} / \mathrm{dl}$ with estimated glomerular filtration rate (eGFR) of $12 \mathrm{ml} / \mathrm{min} / 1.73 \mathrm{~m}^{2}$ which were found worsened on this admission with creatinine 6.4 and urea 201 with eGFR $9 \mathrm{ml} / \mathrm{min} / 1.73 \mathrm{~m}^{2}$. Complete hemogram revealed hemoglobin $7.1 \mathrm{~g} / \mathrm{dl}$ microcytic hypochromic, total leukocyte count of $6800 / \mathrm{mm}^{3}$, and platelet count of $240,000 / \mathrm{mm}^{3}$. Liver function tests were unremarkable with albumin of $3.6 \mathrm{~g} / \mathrm{dl}$. Uric acid was $9.6 \mathrm{mg} / \mathrm{dl}$, calcium was $7.9 \mathrm{mg} / \mathrm{dl}$, phosphorus was $8.9 \mathrm{mg} / \mathrm{dl}$, and normal arterial blood gas analysis. Erythrocyte sedimentation rate was $24 \mathrm{~mm} / \mathrm{h}$, Mantoux test was negative, and RA factor was negative. Digital chest $\mathrm{X}$-ray revealed left-sided pleural effusion with right-sided tracheal shift with clear costophrenic angle on the right side (Fig. 1a).

On thoracentesis, the pleural fluid was hemorrhagic, exudative with pleural fluid-to-serum protein ratio was $0.54(3.5 / 6.4)$, and the pleural fluid to serum lactate dehydrogenase ratio $>0.6(294 / 254)$ with lymphocytic predominance and adenosine deaminase of 9.1 . No organisms were seen on Gram stain, no acid-fast bacilli were seen, and the cartridge-based nucleic acid amplification test was negative. High-resolution computed tomography chest showed massive left-sided pleural effusion with no evidence of enlarged lymph nodes and no infiltrates (Fig. 2). No malignant cells were

Correspondence to: Karthik Ambalavana, Department of General Medicine, Pandit Bhagwat Dayal Sharma Post Graduate Institute of Medical Sciences, Rohtak, Haryana, India. E-mail: karthikambalavana2000@gmail.com

(C) 2021 Creative Commons Attribution-NonCommercial 4.0 International License (CC BY-NC-ND 4.0). 

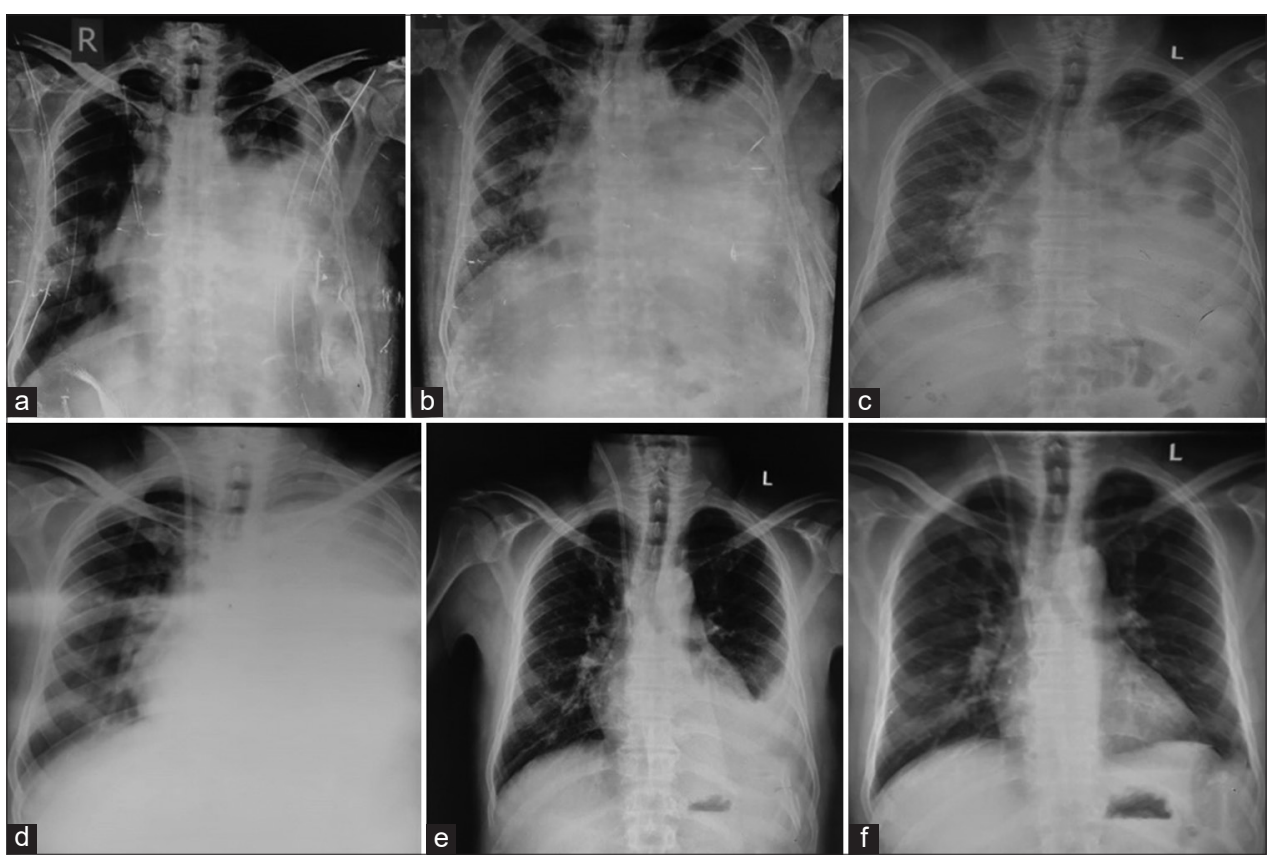

Figure 1: Digital chest X-ray posterior-anterior (PA) view (a) on admission; (b) after diagnostic thoracocentesis; (c) after therapeutic thoracocentesis. Digital chest X-ray PA view with central venous catheter in situ (d) after second therapeutic thoracocentesis; (e) after two sessions of hemodialysis; (f) on follow-up visit

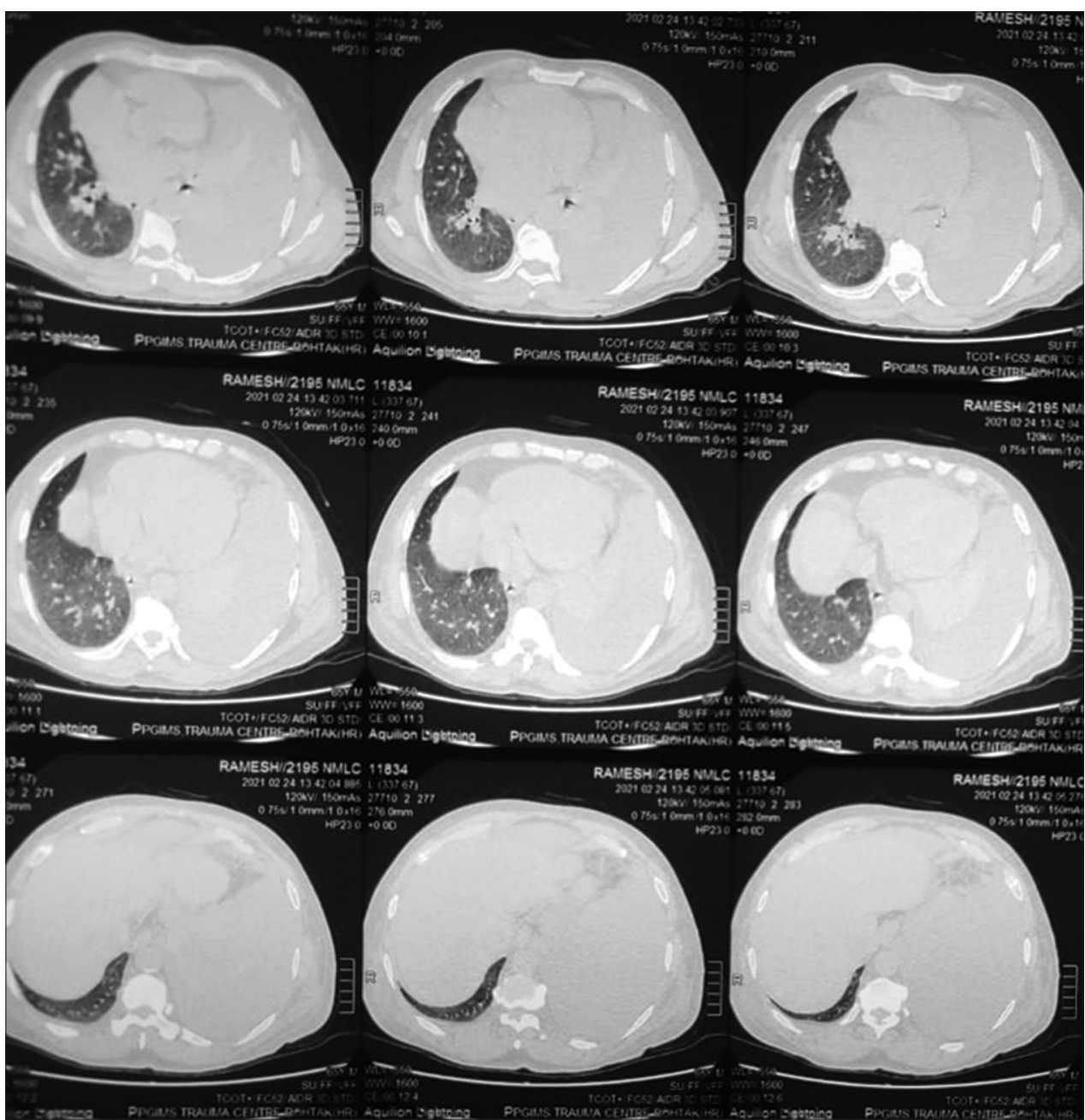

Figure 2: High-resolution computed tomography thorax showing massive pleural effusion with underlying collapse in the left lung with tracheal deviation toward the right 
noticed on pleural fluid cytological examination done on two separate samples.

The patient was started on diuretics, empirical antibiotics, and other supportive treatments but the shortness of breath worsened. A repeat chest X-ray revealed a massive pleural effusion (progressed from before) (Fig. 1b). Therapeutic thoracentesis was done which gave symptomatic relief (Fig. 1c). However, after 2 days, the patient again started complaining of shortness of breath which progressed over the next 2 days, and hence, a repeat thoracentesis was done. No resolution was noticed on the chest X-ray (Fig. 1d).

The patient was planned for pleural biopsy but the patient denied to give consent for the same. Therefore, keeping uremic effusion as a possibility, hemodialysis was done. After two sessions, the patient improved symptomatically, shortness of breath reduced gradually, and saturation at room air was $97 \%$. A resolution was noticed on the chest X-ray (Fig. 1e) and two more sessions of hemodialysis were done. The patient was discharged in hemodynamically stable condition and further hemodialysis was withheld as the patient was having stable renal parameters and adequate urine output (Fig. 1f).

\section{DISCUSSION}

Pleural effusion is a common complication in patients with $\mathrm{CKD}$. The incidence of pleural effusion has been reported to be approximately $3 \%$ in end-stage renal disease (ESRD) cases and no correlation was found between the severity of disease and the presence of pleural effusion. Transudative pleural effusion is caused by hypervolemia, whereas, exudative pleural effusion is caused secondary to various etiologies such as infections, malignancies, and rarely uremia. Hypervolemia and heart failure are the most common causes. Uremia is a rare cause of pleural effusion.

The pathogenesis of uremic pleuritis remains uncertain and it has primarily been reported in patients with ESRD receiving dialysis. Uremic pleuritis is a diagnosis of exclusion resulting from inflammation of the visceral and parietal pleura [1].

Only a few cases of CKD-associated pleural effusion have been reported in patients during the pre-dialysis stage. In our patient, the pleural effusion appeared before the initiation of maintenance hemodialysis. It was unilateral and exudative in nature. With the above-mentioned presentation, a multitude of differential diagnoses was kept in mind and each one was ruled out accordingly before arriving at our final diagnosis. Secondary etiologies such as tuberculosis, malignancies, parapneumonic effusion, pulmonary thromboembolism, and connective tissue diseases were ruled out [2-4]. The pleural effusion improved after dialysis, and hence, the diagnosis of uremic effusion was inferred. Therefore, we came to a conclusion that an inflammatory process such as uremic pleuritis could cause exudative pleural effusion, although we could not confirm this case as uremic pleuritis due to the lack of pleural biopsy. Uremic effusion should be considered as a diagnosis of exclusion, and as in our case, there was a resolution of effusion after multiple sessions of dialysis, the diagnosis of pleural effusion secondary to uremia was considered in our patient.

Usually, half of the patients are asymptomatic, while the remaining patients present with fever, pleuritic chest pain, dyspnea, or non-productive cough [5]. A close relationship between the degrees of uremia and the occurrence of pleural effusion has not been found.

The treatment options available for patients with uremic pleural effusion are intensified continued hemodialysis, repeated thoracentesis, chest tube drainage, pleurodesis, and surgical pleurectomy [6]. Pleural effusion in our patient responded to hemodialysis. However, the patients may be unresponsive to these therapies. Corticosteroids have even been tried in some refractory cases [7-9]. But unfortunately, to date, the optimal treatment in refractory cases has not been established. Seo et al. reported a case of refractory exudative pleural effusion in patients with CKD not receiving dialysis [10].

\section{CONCLUSION}

Unilateral pleural effusion is a diagnostic challenge in CKD patients. Uremia should be considered as a cause of exudative unilateral pleural effusion after other most common causes of pleural effusion such as tuberculosis and parapneumonic effusions are ruled out. Establishing the cause is necessary in view of its therapeutic implications.

\section{REFERENCES}

1. Maher J. Uremic pleuritis. Am J Kidney Dis 1987;10:19-22.

2. Berger HW, Rammohan G, Neff MS, Buhain WJ. Uremic pleural effusion. A study in 14 patients on chronic dialysis. Ann Intern Med 1975;82:362-4.

3. Ray S, Mukherjee S, Ganguly J, Abhishek K, Mitras S, Kundu S. A cross sectional prospective study of pleural effusion among cases of chronic kidney disease. Indian J Chest Dis Allied Sci 2013;55:209-13.

4. Kumar AP, Pathrudu BS, Rani NU. A study on etiology on etiology and profile of pleural effusion in chronic kidney disease. J Evol Med Dent Sci 2015;5:11785-96.

5. Isoda K, Hamamato Y. Uremic pleuritis. Clinicopathologic analysis of 26 autopsy cases. Bull Osaka Med Sch 1984;30:73-80.

6. Bintcliffe OJ, Lee GY, Rahman NM, Maskell NA. The management of benign non-infective pleural effusions. Eur Respir Rev 2016;25:303-16.

7. Iyoda M, Ajiro Y, Sato K. A case of refractory uremic pleuropericarditissuccessful corticosteroid treatment. Clin Nephrol 2006;65:290-3.

8. Kim KM, Cho JM, Park HJ. A case of refractory uremic pleuritis improved completely with corticosteroid treatment. Clin Nephrol 2008;70:451-2.

9. Park EJ, Park MA, Park MJ, Park SY, Lee SH. Corticosteroid therapy for refractory uremic pleurisy. Ewha Med J 2016;39:125-8.

10. Seo HM, Kim M, Kim H. Refractory exudative pleural effusion in patients with chronic kidney disease not receiving dialysis: A case report. Clin Case Rep 2019;7:675-9.

Funding: None; Conflicts of Interest: None Stated.

How to cite this article: Atri SK, Ambalavana $\mathrm{K}$, Dhanda A, Kumar S. Unilateral pleural effusion in chronic renal insufficiency: A clinician's challenge. Indian J Case Reports. 2021;7(6):238-240. 